rative and sedative effects which were required. Saline draughts were given frequently, free sponging of the body was used with much advantage, and toast and barley-water were supplied liberally for drink. Diet to be low. The most usual complication was a cerebral affection. Cold applications were constantly renewed to the head, which was often shaved. A blister to the nape of the neck was extremely efficacious, if the case became urgent. At the end of the first week after admission, a kind of crisis appeared. After several hours' profuse perspiration, the patient's symptoms decidedly improved; the tongue began to clean, the pains in the limbs to abate, and all that was complained of was extreme weakness. More substantial diet seemed to be indicated, and in another day or two the patient would earnestly request it. If the symptoms continued to improve under more nourishing regimen, as in the majority of instances was the case, for a few days all promised exceedingly well; but almost invariably, by the end of another week, a sudden change would take place, the tongue become as coated as ever, and all the original symptoms would present themselves, and apparently in as much force as at first. The recurrence to low diet of course immediately followed: the relapse was treated as the primary attack, though usually it proved less obstinate, in point of duration. The recovery would then take place very slowly. The longest remaining sequence was want of muscular power, and most patients, though looking quite well, found, on their dismission from the Infirmary, a much greater prostration of the vital powers than either they or any casual observer would have suspected. None, I should think, were able to engage in agricultural labour during the season. At the end of the first week reforred to above, just as the system was throwing off the first onset of the febrile attack, when the arterial action was diminishing, no local congestions presenting, and the digestive functions were seemingly in more healthy condition, the crisis would frequently result in a different course. After two or three days of apparent quiescence, which might be presumed, on the whole, to be something like general improvenent, a yellowish line would appear down the centre of the tongue; dryness and a slightly cracked appearance followed; the pulse became weaker, and gradually sank to 60 or 70 ; the countenance wore a more anxious expression; and there was disinclination to food. On the administration of port wine or brandy, as the state of the case might seem to indicate, these symptoms would perhaps gradually subside, and the recovery might take place withont the relapse, as in the foregoing instances; but in a few cases, complete typhus ensued, with the usual symptoms of great nervous depression. These were constantly treated with concentrated nourishment and stimulants, and when the power of swallowing was in aberance, strong beef-tea injections supported Nature until her powers rallied. Ammonia and quinine, with small opiates, were administered according to circumstances; and with close watching and constant attention, the third week would nearly bring the patient through the severity of the attack, which then left him in an exceedingly weak condition. Here especially-although the rule applies generally to every case-the greatest care was necessary in directing the advance towards convalescence. That it should be sure, it was absolutely necessary to be also slow.

In reviewing the general character of the fever in question, it is worthy of remark, that the complications were those of the head and the mucous lining of the intestinal canal. To three patients (out of 179) jaundice occurred, terminating fatally in one case. In one or two instances only was there any tendency to chest affection, as a consequence of the fever. From these details, it will be apparent that the form of fever described approaches nearly to that known as the "simple continued," the "Synochus of Callen." But a strong tendency invariably existed towards a termination in one of a low nervous type or typhoid. Keeping this in view, the indications were plain-viz, to be as sparing of antiphlogistic treatment as the case would allow, discarding the use of the lancet altogether. Even in the administration of mercury, antimony, and purgative medicines, great care was necessary, not to be too liberal in the use of depressants, and to commence the exhibition of nutritious diet, however small the quantity, as soon as the stomach was enabled to perform its functions. The first tendency to exhaustion was promptly met by stimulants cautiously and gradually afforded, according to the urgency of the symptoms, with strict attention to the cerebral affection, as evidenced by a comatose condition of the patient, insensibility of the pupil, subsultus tendinum, \&c., which were combated by means of cold applications and counter-irritation. With unremitting attention to ventilation and cleanliness I believe little or no danger of infection existed, and the patients were distributed indiscriminately among those suffering from other diseases. Had separate wards been devoted to the fever patients alone, I have no doubt the results would have been far less favourable; under the plan adopted the other patients have not suffered. Two out of six nurses who were engaged in constant attendance were attacked, and exhibited the type of disease described, in rather a severe form, but this is by no means surprising if we consider the very arduous character of their engagements at this period. I acted on the principle of providing full and stimulating diet for them, and I recommended to the visiting clergymen the only precaution which I and those who assisted me adopted -namely, the taking a glass of wine and a biscuit before entering the wards. Not the slightest fever symptoms were manifested in those brought into contact with the sick.

"I have no hesitation in attributing so small a mortality4 in 179 -chiefly to the unlimited supply of stimulus and concentrated nutriment which $I$ had it in my power to afford, and to the constant exertions and watchful attention with which the much-respected matron of the Infirmary and our experienced nurses seconded the directions of the medical at tendants."

\section{Description of an Improved Tourniquet.}

By H. V. MLALAN, M.D.

Dr. Malan urges his claim as the inventor of an improved tourniquet, now in use in Great Britain and on the Contivent, and he subjoins a description of the instrument, as given in his "Dissertation for Obtaining the Diplomas of Doctor of Medicine, Surgery, and Midwifery, in the University of Tubingen, in 1839." This tourniquet, he states, was then publicly examined and tried before competent judges, and a description of it was soon afterwards published in a medical journal, under the sanction of the rector of Tubingen University.

Dr. Malan states-" During my subsequent travels I found it lectured upon in Paris, Lyons, and Edinburgh, by various professors of surgery. My friend the late Mr. Liston was in the habit of using it.

"Finding it, therefore, known and valued in the profession, I feel anxious not to allow it to remain unconnected with my name; and the more so, as it is frequently mentioned in medical publications and journals, without the acknowledgments ordinarily due to the inventor."

A technical description of "Malan's tourniquet" accompanies Dr. Malan's communication; but it would be unsuitable in thi - place, and without many attendant engravings. The chief advantages of the instrument are stated to be these:-

"As the key can be adapted to either end of the instrument, it can be placed on either side of the limb, below or above, as required. The instrument, as a single glance will prove, is simple and strong; it can be put on, fixed, and tightened, with littie trouble or effort on the part of the operator, and, owing to its two double screws, with much power and rapidity; the compression is especially made on the bloodvessel, by means of the pad, and its very construction makes it lie low and close to the limb, so that not only it cannot be upset by the sudden collapse of the muscles after the amputation, but can be used in any position or applied to any part of the body, and is not in the way of any garment slipped over it; it is compact, small, and safe. As the key may be remored when the instrument is once fixed, no patient can, in any case, free himself from its grasp. Such, $I$ believe, is the general opinion, since it has been in use, now upwards of eight years.

Upper Seymour-street West, Portman-square."

\section{3ncbieins.}

Medicine an Art, and its Truths to be Attained. By Thoras Shaprer, M.D., \&c. London: Churchill. 1848. Pamphlet. pp. 31 .

THIS is an Address read recently at the opening meeting of the Library of the Exeter Dispensary, and of the Devon and Exeter Pathological Society. The writer, using Dr. Whewell's definition, places medicine among the arts -and indeed it will be admitted by all that practical medicine is an art, however, we believe we may, in another point of view, deem medicine a science. The greater part of this address is taken up by a consideration of the fallacies committed 